\title{
Mechanisms for an abnormal radionuclide left ventricular ejection fraction response to exercise in patients with chronic, severe aortic
} regurgitation

\begin{abstract}
To clarify the mechanisms for an abnormal radionuclide left ventricular (LV) ejection fraction response to exercise in patients with chronic, severe aortic regurgitation (AR), we studied seven control patients and 21 patients with AR. We used exercise radionuclide angiography and catheterization of the right and left sides of the heart to obtain a calculation of LV chamber elastance. The control and AR groups had similar heart rates, systolic blood pressure responses to exercise, and exercise durations. In both patient groups, LV end-diastolic volume did not change with exercise. In contrast to the decrease in LV end-systolic volume $(p<0.05)$ and increase in LV ejection fraction $(p<0.01)$ in the control group, $L V$ end-systolic volume in the patients with AR increased, resulting in little change in their LV ejection fraction. By stepwise multiple regression analysis, the radionuclide $L V$ ejection fraction at peak exercise in patients with AR was determined by the LV chamber elastance, LV end-systolic volume, and stroke volume at peak exercise (cumulative $r=0.79, p<0.02$ ); the change in radionuclide $L V$ ejection fraction from rest to peak exercise was determined by the corresponding change in systemic vascular resistance, regurgitant index, and LV end-diastolic and end-systolic volumes (cumulative $r=0.88$, $p<0.02$ ). These data demonstrate that in patients with AR, the radionuclide $L V$ ejection fraction at peak exercise is principally determined by the cumulative effects of chronic, severe AR on LV systolic chamber performance, and the change in radionuclide LV ejection fraction from rest to peak exercise is principally established by peripheral vascular responses. (AM HEART J $1992 ; 123: 453$.
\end{abstract}

Richard E. Stewart, MD, Milton D. Gross, MD, and Mark R. Starling, MD. Ann Arbor, Mich.

A noninvasive technique that could follow the sequential changes in left ventricular (LV) size and performance in patients with chronic, severe aortic regurgitation (AR) who have minimal or no symptoms and thus that could guide the selection of patients for cardiac catheterization and aortic valve replacement would be helpful. Exercise radionuclide angiography has been proposed for this purpose. ${ }^{1}$ It has been shown that this noninvasive technique can

From the Divisions of Cardiology and Nuclear Medicine, Department of Internal Medicine, University of Michigan and Veterans Administration Medical Centers.

Supported by National Institutes of Health grants RO1-HL36450 and MO1-RR00042 from the National Heart, Lung, and Blood Institute, Bethesda, Md., and the Veterans Administration, Washington, D.C.

Received for publication May 13, 1991; accepted July $\perp$, 1991.

Reprint requests: Mark R. Starling, MD, Department of Internal Medicine, Division of Cardiology, Veterans Administration Medical Center, 2215 Fuller Rd., Ann Arbor, MI 48105.

4/1/33950 quantitate the changes in LV size and performance between rest and peak exercise. ${ }^{2}$ Moreover, it has been suggested that this technique may be useful for identifying those who will have progression of their symptoms and who will need aortic valve replacement among patients with $\mathrm{AR}$ who have minimal or no symptoms. ${ }^{3}$

The precise pathophysiologic mechanism responsible for an abnormal radionuclide LV ejection fraction response to exercise in these patients is, however, a controversial topic. ${ }^{4-7}$ Some believe that an abnormal radionuclide $L V$ ejection fraction response to exercise in patients with AR is related to loading conditions $^{5}$ or the peripheral vascular changes that occur with exercise. ${ }^{6}$ In contrast, other investigators have suggested that the chronic effects of AR on LV systolic performance or contractility play an integral role in determining how the left ventricle responds to exercise. ${ }^{7}$ This investigation was designed to clarify the mechanisms for an abnormal radionuclide $L V$ ejection fraction response to exercise in patients with 
Table I. Baseline hemodynamic values

\begin{tabular}{lccc}
\hline & Controls $(n=7)$ & Aortic regurgitation $(n=21)$ & $p$ Value \\
\hline HR & $71 \pm 7$ & $71 \pm 8$ & N.S. \\
SBP (mm Hg) & $143 \pm 22$ & $147 \pm 25$ & N.S. \\
RAP (mm Hg) & - & $7 \pm 3$ & - \\
PCWP (mm Hg) & - & $12 \pm 6$ & - \\
LVEDP (mm Hg) & $12 \pm 4$ & $18 \pm 10$ & N.S. \\
LVSP (mm Hg) & $129 \pm 32$ & $143 \pm 30$ & N.S. \\
+dP/dt max (mm Hg/sec) & $1377 \pm 442$ & $1239 \pm 327$ & N.S. \\
$\mathrm{E}_{\max }(\mathrm{mm} \mathrm{Hg/ml)}$ & $4.94 \pm 1.14$ & $1.82 \pm 1.24$ & $<0.001$ \\
\hline
\end{tabular}

$H R$, Heart rate; $S B P$, systolic blood pressure; $R A P$, right atrial pressure; $I C W P$, pulmonary capillary wedge pressure; $L V E D P$, left ventricular end-diastolic pressure; $L V S P$, left ventricular systolic pressure; $+d P / d t$ max, maximum rate of change of LV pressure; $E_{\max }$, chamber elastance.

chronic, severe AR and therefore to possibly lead to an understanding of why an abnormal response to exercise is predictive of symptomatic and hemodynamic deterioration.

\section{METHODS}

Patients. The patient population consisted of seven patients in the control group and 21 patients with chronic, severe AR. The seven patients in the control group were men with an age range of 40 to 72 years (mean $56 \pm 10$ years). No patient in the control group had experienced a myocardial infarction, and all had normal findings on electrocardiograms, chest roentgenograms, and physical examinations. These patients had been referred for cardiac catheterizations so that their chest pain syndromes could be evaluated. They were all found to have normal coronary anatomy, LV volumes, ejection fractions, and wall motion.

The patients with AR consisted of 20 men and one woman with an age range of 33 to 78 years (mean $56 \pm 14$ years). Four patients were in clinical class $I, 11$ were in class II, six were in class III, and no patient was in class IV. Thus the majority of patients with AR had minimal or no symptoms. At the time of referral 14 patients had LV hypertrophy on an electrocardiogram, and eight patients had cardiomegaly on a chest radiograph. These patients had evidence on physical examination of moderate to severe AR. All patients had been referred so that the hemodynamic significance of their AR could be defined. All were found to have normal coronary anatomy, angiographic $3+$ or $4+A R$, and no evidence of significant aortic stenosis (aortic valve gradient of $10 \mathrm{~mm} \mathrm{Hg}$ or less).

Protocol. All patients provided written informed consent on forms approved by the Institutional Review Boards at the University of Michigan or VA Medical Centers. All $\beta$-adrenergic-blocking and calcium-channel-blocking agents, vasodilators, inotropic agents, diuretics, and nitrates were discontinued 24 to 48 hours before the initiation of the studies. The day before the cardiac catheterization, each patient, using a braked bicycle ergometer, underwent a supine bicycle exercise radionuclide angiogram. The work load was begun at 200 kilopond-meters $(\mathrm{kpm})$ and was progressively increased in increments of 100 $\mathrm{kpm}$ every 3 minutes to a symptom limit. Gated equilib- rium radionuclide angiograms were obtained at each patient's rest and peak exercise. On the following day a diagnostic catheterization of the right and left sides of the heart documented resting intracardiac pressures, cardiac output, and normal coronary anatomy. Micromanometer LV pressures and radionuclide angiograms were then obtained under baseline conditions and after methoxamine or nitroprusside infusions to achieve multiple additional loading conditions with right atrial pacing to maintain heart rate constant. $^{8}$

Radionuclide angiography. The gated equilibrium radionuclide angiograms were acquired with the use of a $\mathrm{Si}$ emens Basicam (Siemens Medical Systems Inc., Iselin, N.J.) fitted with a low-energy all-purpose collimator. All studies were obtained after in vivo red blood cell labeling with $30 \mathrm{mCi}$ technetium $99 \mathrm{~m}$ for $30 \mathrm{msec}$ frames throughout the cardiac cycle for 250 cardiac cycles. Midway through each radionuclide acquisition, a $2 \mathrm{ml}$ blood sample was drawn. The blood samples were later counted for 2 minutes, and the time delay between acquisition and counting of the blood samples was recorded. At the completion of exercise or cardiac catheterization, distance measurements were obtained for each patient to determine the $\mathrm{LV}$ geometric center for attenuation correction.

Attenuation-corrected radionuclide $\mathrm{LV}$ volumes were calculated frame by frame by use of background-subtracted, hand-drawn, region-of-interest count data; decaycorrected blood sample counts; and attenuation correction as previously validated in this laboratory. ${ }^{9}$ Right ventricular end-diastolic and end-systolic counts were obtained by use of a modification of the method of Maddahi et al. ${ }^{10} \mathrm{We}$ have used this method to calculate right ventricular (RV) volumes for comparison to those obtained from biplane contrast cineventriculography ${ }^{11}$ and to calculate $R V$ volumes and ejection fractions in patients with RV infarction. ${ }^{12}$ We used these data to calculate $\mathrm{LV}$ regurgitant in$\operatorname{dex}$ (RI) as RI = (LVEDC-LVESC)/(RVEDC-RVESC), where EDC represents end-diastolic counts at maximum ventricular volume and $\mathrm{ESC}$ represents end-systolic counts at minimum ventricular volume for the $L V$ and $R V$ from the hand-drawn regions of interest.

Cardiac catheterization. After coronary arteriography and cineventriculography were performed in the control 
Table II. Exercise hemodynamic values

\begin{tabular}{lccc}
\hline & Controls $(\mathbf{n}=7)$ & Aortic regurgitation $(\mathbf{n}=21)$ & $p$ Value \\
\hline Exercise duration (min) & $12.6 \pm 4.0$ & $12.0 \pm 3.5$ & N.S. \\
Peak HR & $125 \pm 18$ & $127 \pm 20$ & N.S. \\
Peak SBP (mm Hg) & $203 \pm 26$ & $197 \pm 26$ & N.S. \\
Rest LVEDV (m) & $151 \pm 41$ & $303 \pm 183$ & $<0.05$ \\
Peak LVEDV (ml) & $147 \pm 35$ & $313 \pm 182$ & $<0.05$ \\
Rest LVESV (m) & $69 \pm 24$ & $165 \pm 112$ & $<0.05$ \\
Peak LVESV (ml) & $50 \pm 13^{*}$ & $177 \pm 129$ & $<0.05$ \\
Rest LVEF (c) & $53 \pm 7$ & $50 \pm 11$ & N.S. \\
Peak LVEF (co) & $63 \pm 7^{* *}$ & $48 \pm 12$ & $<0.01$ \\
Rest CO (L/min) & $5.8 \pm 1.7$ & $5.6 \pm 1.6$ & N.S. \\
Peak CO (L/min) & $11.5 \pm 2.5^{* *}$ & $10.6 \pm 6.9^{* *}$ & N.S. \\
Rest SV (ml) & $81+18$ & $73+36$ & N.S. \\
Peak SV (ml) & $97 \pm 27$ & $92 \pm 58$ & N.S. \\
Rest RI & - & $2.5 \pm 1.9$ & - \\
Peak RI & - & $2.1 \pm 1.1$ & - \\
Rest SVR (dynes/sec-cm & $1870 \pm 889$ & N.S. \\
Peak SVR (dynes/sec-cm $\left.{ }^{5}\right)$ & $1242 \pm 365$ & $1426 \pm 966$ & N.S. \\
\hline
\end{tabular}

HR, Heart rate; SBP, systolic blood pressure; LVESV, left ventricular end systolic volume; LVEF, left ventricular ejection fraction; CO, cardiac output; $\mathrm{SV}$, effective stroke volume; RI, regurgitant index; SVR, systemic vascular resistance.

$* * p<0.01$ vs rest values.

group and additional ascending aortography was performed in the patients with AR, a bipolar pacing catheter was placed into the right atrium of each patient to maintain heart rate constant, and a precalibrated micromanometer catheter (Millar Instruments, Houston, Texas) was positioned to measure $L V$ pressure. Hemodynamic recordings were obtained with an Electronics for Medicine VR12, (PPG Biomedical Systems, Lenexa, Kan.) or Micor physiologic recorder, (Micor, Inc. Allison Park, Pa.) at 100 $\mathrm{mm} / \mathrm{sec}$ paper speed. They included the simultaneous recording of an electrocardiographic lead (II), micromanometer $\mathrm{LV}$ and aortic pressure, and the first derivative of $\mathrm{LV}$ pressure $(\mathrm{dP} / \mathrm{dt})$. These hemodynamic recordings were obtained for 10 to 20 cardiac cycles at the beginning, middle, and end of each radionuclide acquisition.

The LV pressure waveforms were averaged and the average LV pressure waveforms were hand digitized with a Calcomp 9100 inductance-digitizing surface (resolution $0.02 \mathrm{~mm}$ ) (Calcomp, Anaheim, Calif.) interfaced to an IBM XT, (IBM Corp., Armonk, NY.) beginning at the peak of the $\mathrm{R}$ wave of the simultaneously recorded electrocardiogram. ${ }^{8}$ This program yields instantaneous $\mathrm{LV}$ pressure and the first derivative of $\mathrm{LV}$ pressure, $\mathrm{dP} / \mathrm{dt}$, at a variable sampling frequency. Interpolation of these hand-digitized LV pressure data was then performed to obtain instantaneous LV pressures that coincided with the midpoint of each radionuclide frame so that isochronal $L V$ pressure and volume data were available for further analysis.

Calculation of maximum time-varying elastance and other derived hemodynamic parameters. The micromanometer LV pressures were matched to their corresponding radionuclide $L V$ volumes for each loading condition and then plotted to obtain multiple pressure-volume loops. The $\mathrm{LV}$ maximum time-varying elastance $\left(\mathrm{E}_{\max }\right)$ was defined as the maximum slope obtained from the linear regression of isochronal pressure-volume data points from each pressure-volume loop. $E_{\max }$ has been proposed as a relatively load-independent index of contractility. ${ }^{13}$ This is probably valid when $\mathrm{E}_{\max }$ is measured in the same heart after pharmacologic interventions, which either positively or negatively affect contractility. ${ }^{14,15}$ However, when $\mathrm{E}_{\max }$ is calculated in different hearts, it may be affected by several factors other than contractility. ${ }^{16,17}$ Accordingly, in this investigation $E_{\max }$ is used to represent net $L V$ systolic chamber performance.

Several hemodynamic parameters were calculated from the radionuclide data at rest and peak exercise. The "effective" LV stroke volume was calculated as $\mathrm{SV}=(\mathrm{LVEDV}-\mathrm{LVESV}) / \mathrm{RI}$, where EDV and ESV represent radionuclide $\mathrm{LV}$ end-diastolic and end-systolic volumes and RI represents LV regurgitant index. "Effective" is used in this context as that portion of the total LV stroke output that does not contribute to regurgitant volume and thus would be effectively perfusing the peripheral tissues. Cardiac output (CO) was then calculated as $\mathrm{CO}=$ $\mathrm{SV} \times \mathrm{HR}$, where SV represents "effective" LV stroke volume and HR represents heart rate. The systemic vascular resistance $(\mathrm{SVR})$ was calculated as $\mathrm{SVR}=(\mathrm{mBP} / \mathrm{CO}) \times 80$ dynes/sec-cm ${ }^{5}$, where $\mathrm{mBP}$ represents mean brachial artery pressure determined as diastolic blood pressure (BP) plus one third of (systolic BP - diastolic BP).

Statistical analysis. All data are expressed as the mean $\pm 1 \mathrm{SD}$. Nonpaired $t$ tests were used to compare the rest and peak exercise and the change in hemodynamic parameters from rest to peak exercise between the control patients and patients with AR. Paired $t$ tests were used to compare the LV size and performance measures at rest and peak exercise within each patient group. Correlation ma- 


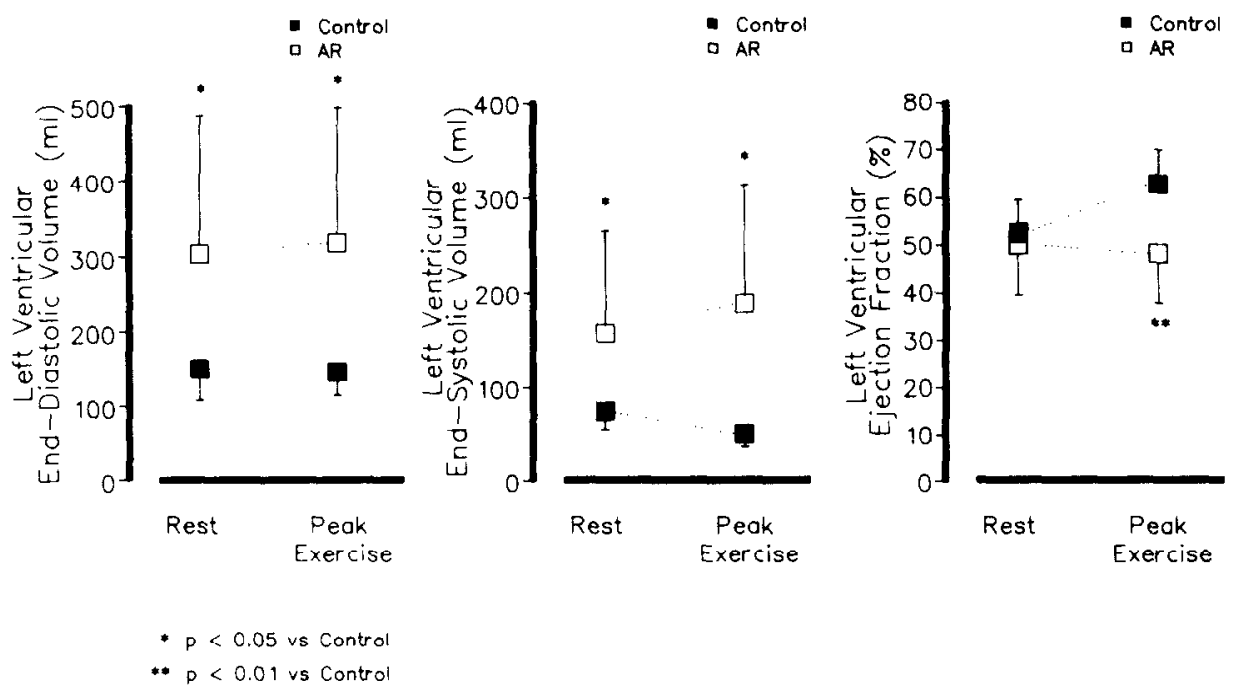

Fig. 1. The average LV end-diastolic volumes (left panel), end-systolic volumes (center panel), and ejection fractions (right panel) for the control groups (closed boxes) and the patients with chronic, severe AR (open boxes) are shown at rest and peak exercise. Significant differences between the rest and peak exercise values for the control and AR groups are noted.

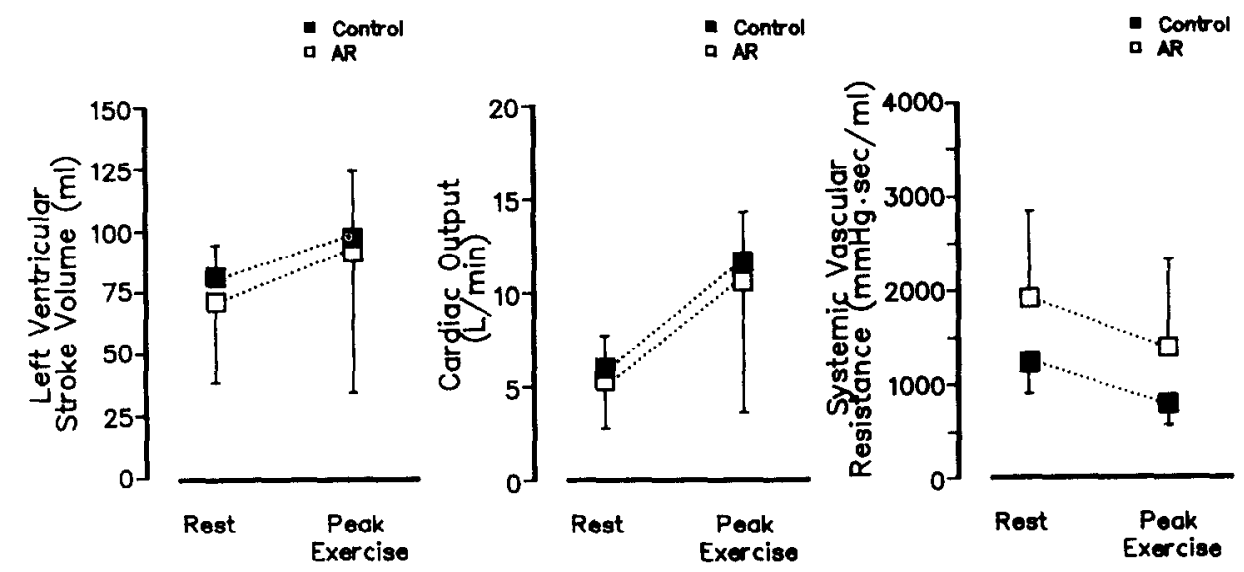

Fig. 2. The average rest and peak exercise values for the control patients and patients with chronic, severe AR are shown for "effective" stroke volume (left panel), cardiac output (central panel), and systemic vascular resistance (right panel). The format is similar to Fig. 1, and significant differences are noted.

trices and stepwise multiple regression analyses were then performed to determine those hemodynamic parameters that independently determine the peak exercise radionuclide $\mathrm{LV}$ ejection fraction and the change in radionuclide $\mathrm{LV}$ ejection fraction from rest to peak exercise. A $p$ value of 0.05 or less was considered significant.

\section{RESULTS}

Baseline hemodynamic data. At the time of cardiac catheterization, 10 patients with AR had angiographic $+4 \mathrm{AR}$ and the remaining 11 patients had +3 $A R$. The heart rate averaged $71 \pm 7$ beats $/ \mathrm{min}$ and the right atrial and pulmonary capillary wedge pressures averaged $7 \pm 3$ and $12 \pm 6 \mathrm{~mm} \mathrm{Hg}$, respec- tively. The mean cardiac output was $5.6 \pm 1.6 \mathrm{~L} / \mathrm{min}$. The micromanometer LV end-diastolic and end-systolic pressures averaged $18 \pm 10$ and $143 \pm 30 \mathrm{~mm}$ $\mathrm{Hg}$, respectively, and the average $(+)$ maximum $\mathrm{dP} / \mathrm{dt}$ was $1239 \pm 327 \mathrm{~mm} \mathrm{Hg} / \mathrm{sec}$. The $\mathrm{LV} \mathrm{E} \mathrm{E}_{\max }$ averaged $1.82 \pm 1.24 \mathrm{~mm} \mathrm{Hg} / \mathrm{ml}$, and it was less than that in the control group $(4.94 \pm 1.14 \mathrm{~mm} \mathrm{Hg} / \mathrm{ml}$, $p<0.001$, Table I).

Exercise hemodynamic data. The mean rest and peak exercise hemodynamic data for the control and AR groups are shown in Table II, and they are compared in Figs. 1 and 2. The average heart rate, systolic blood pressure, and pressure-rate product at rest and 


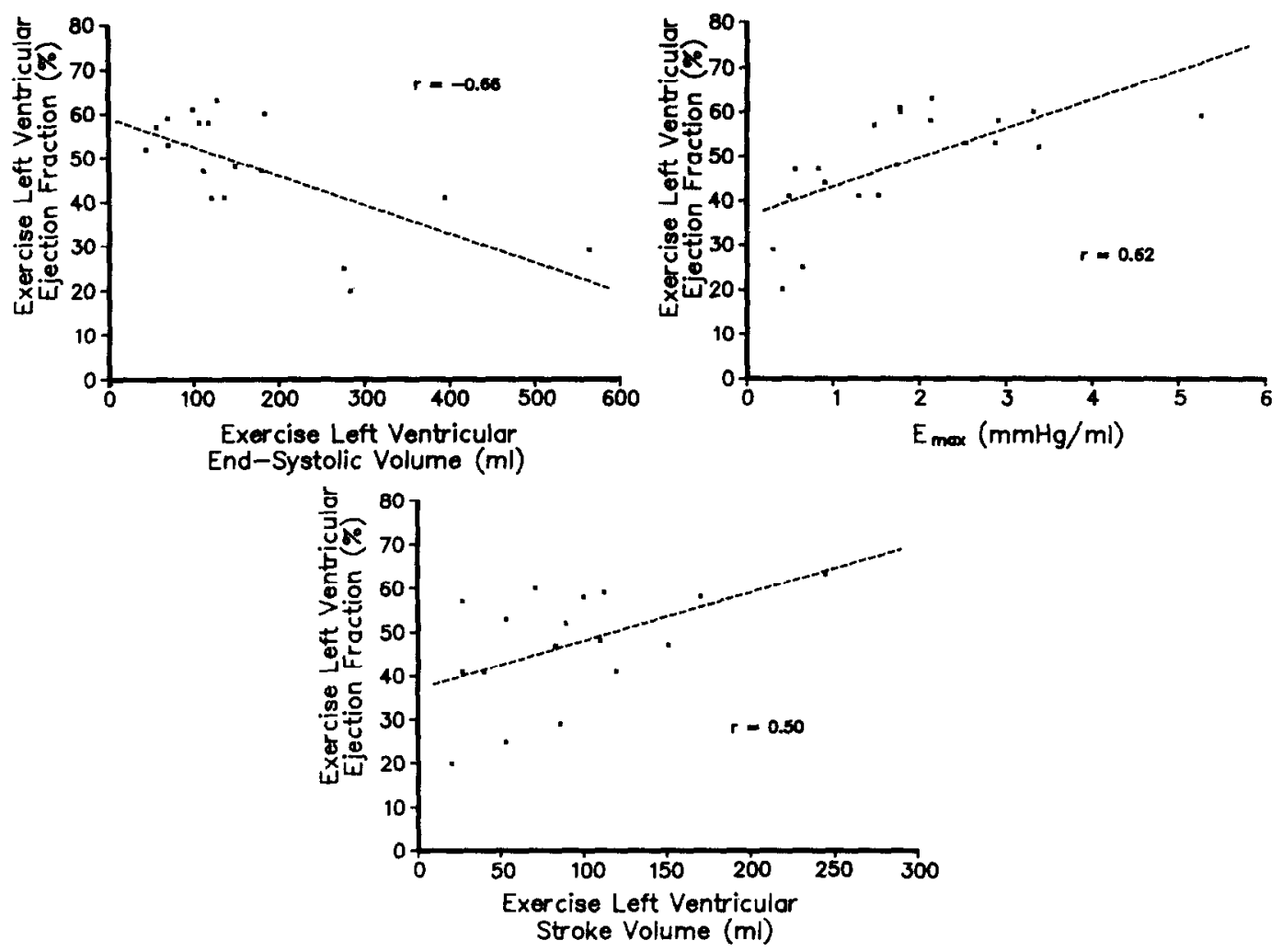

Fig. 3. The relationship between the radionuclide $\mathrm{LV}$ ejection fraction at peak exercise and the corresponding peak exercise LVESV (upper left panel), LV chamber elastance $\left(E_{\text {max }}\right.$, upper right panel), and "effective" stroke volume (lower left panel) are shown. The individual data points, regression lines, and correlation coefficients are noted.

peak exercise in the control and AR groups did not differ significantly. Consequently, the changes in these values did not differ between the two patient groups. The average exercise duration in the two groups were similar.

The LV end-diastolic and end-systolic volumes (LVEDV and LVESV, respectively) were larger in the AR group than in the control group, both at rest ( $p<0.05$ for both) and at peak exercise $(p<0.05$ for both). The changes in LVEDV in the two patient groups were similar. In contrast, the changes in LVESV in the control group differed from those in the AR group because LVESV decreased in the control group ( $p<0.05$ for both) and increased in the AR group. Consequently, although there was no difference in $\mathrm{LV}$ ejection fraction at rest, it differed for each group at peak exercise $(p<0.01)$. This occurred because there was a significant increase in $L V$ ejection fraction $(p<0.01)$ in the control group, but there was no change in $\mathrm{LV}$ ejection fraction from rest to peak exercise in the AR group (Fig. 1).

In the patients with $\mathrm{AR}$ the regurgitant index at rest averaged $2.5 \pm 1.9$. It decreased slightly, but it was statistically unchanged, at peak exercise
$(2.1 \pm 1.1)$. Although the cardiac output was somewhat less in the AR group than in the control group at rest, it did not differ significantly between the groups. In both patient groups, cardiac output increased significantly at peak exercise $(p<0.01$ for control and $p<0.01$ for AR). Consequently, the change in cardiac output was similar in both patient groups. The systemic vascular resistance was higher in the AR group than in the control group at rest $\left(1870 \pm 889\right.$ vs $1242 \pm 365$ dynes-sec/cm $\left.{ }^{5}\right)$, and values continued to differ at peak exercise (1426 \pm 966 vs $824 \pm 183$ dynes-sec $/ \mathrm{cm}^{5}$ ). In the control patients there was a decrease in systemic vascular resistance from rest to peak exercise $(p<0.05)$, while there was a small but insignificant decrease in systemic vascular resistance in the patients with AR (Fig. 2).

Determinants of $L V$ ejection fraction response to exercise. To establish the determinants of the peak exercise $L V$ ejection fraction and the change in $L V$ ejection fraction from rest to peak exercise, a correlation matrix was established for each radionuclide $\mathrm{LV}$ ejection fraction parameter. As shown in Fig. 3, the peak exercise radionuclide $L V$ ejection fraction correlated with LVESV at peak exercise $(r=-0.66$, 

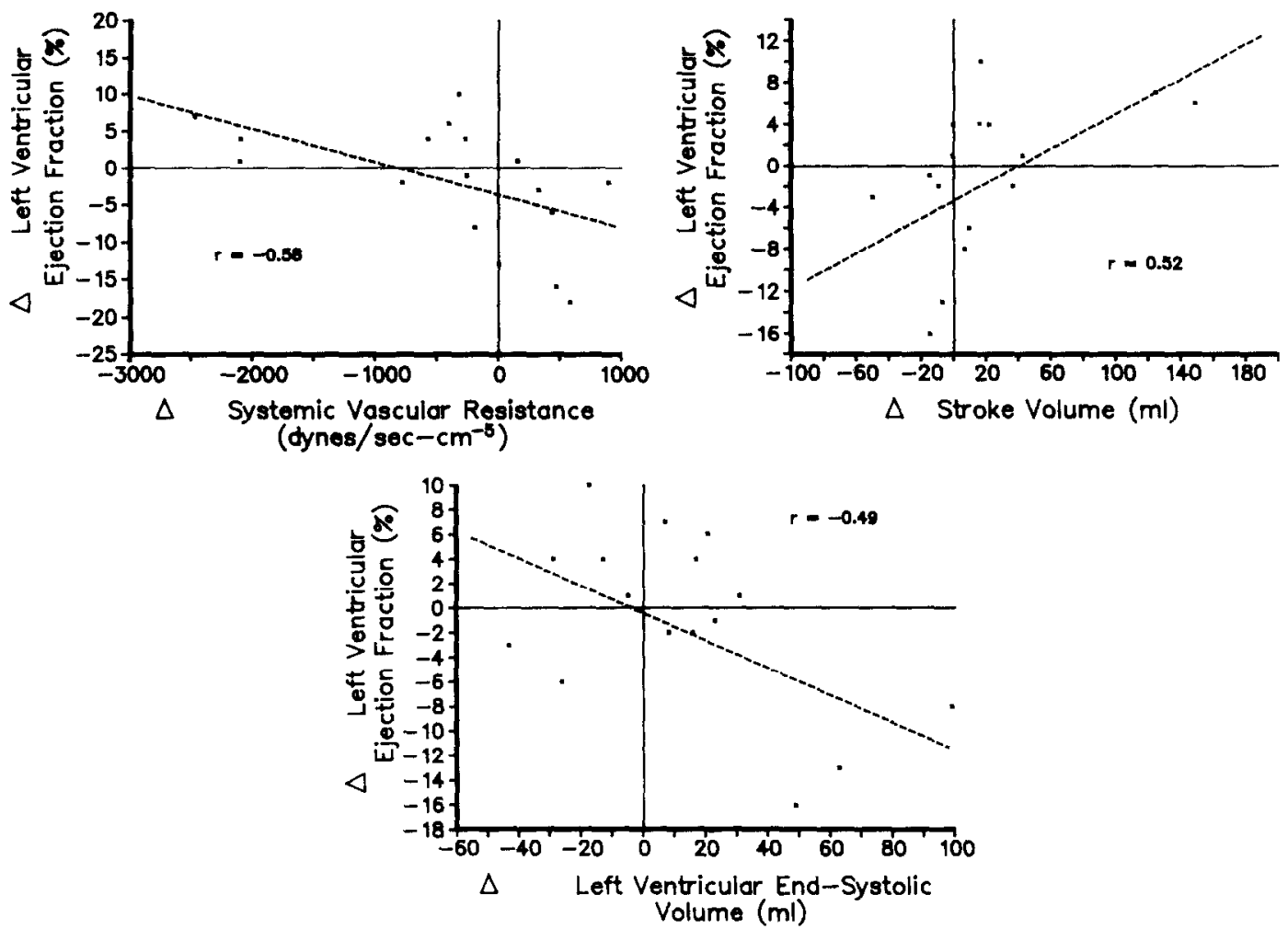

Fig. 4. Similar to Fig. 3 , the change in radionuclide LV ejection fraction from rest to peak exercise and the change in systemic vascular resistance (upper left panel), stroke volume (upper right panel), and LVESV (lower left panel) are shown. The individual data points, regression lines, and correlation coefficients are noted.

$p<0.01), \mathrm{E}_{\max }(r=0.62, p<0.01)$, and "effective" stroke volume at peak exercise $(r=0.50, p<0.05)$. As shown in Fig. 4, the change in LV ejection fraction from rest to peak exercise correlated with the change in systemic vascular resistance $(r=-0.58, p<0.02)$, "effective" stroke volume $(r=0.52, p<0.05)$, and LVESV $(r=-0.49, p<0.05)$. The change in systematic vascular resistance also correlated with the change in regurgitant index $(r=0.63, p<0.01)$.

To establish the independent determinants of the LV ejection fraction at peak exercise and the change in $L V$ ejection fraction from rest to peak exercise, stepwise multiple regression analyses were performed. The $L V$ ejection fraction at peak exercise was determined by $\mathrm{E}_{\max }$, LVESV, and stroke volume at peak exercise (cumulative $r=0.74, p<0.02$ ). The change in LV ejection fraction from rest to peak exercise was determined by the corresponding change in systemic vascular resistance, $\mathrm{LV}$ regurgitant index, and LVEDV and LVESV (cumulative $r=0.88$, $p<0.02$ ).

\section{DISCUSSION}

Borer et al. ${ }^{1}$ originally observed that during exercise the radionuclide $\mathrm{LV}$ ejection fraction increased from $57 \%$ to $71 \%$ in their control group, whereas it decreased from $47 \%$ to $38 \%$ in patients with AR who had symptoms, and it decreased from $62 \%$ to $57 \%$ in patients with AR who had no symptoms. He suggested that the radionuclide $L V$ ejection fraction response to exercise might aid in the detection of LV dysfunction before symptoms and resting $L V$ dysfunction developed and therefore might prove useful for the sequential evaluation of LV performance in these patients. In patients with chronic, severe AR who have no symptoms, the radionuclide $L V$ ejection fraction response to exercise has subsequently been proved useful for predicting the eventual development of symptoms and the need for aortic valve surgery. ${ }^{3}$ The mechanism for an abnormal radionuclide LV ejection fraction response to exercise has, however, remained controversial.

Schuler et al. ${ }^{18}$ calculated the $\mathrm{LV}$ end-systolic pressure-volume relationship using radionuclide angiography in nine patients in a control group and 14 patients with AR, and compared it with the change in radionuclide $L V$ ejection fraction from rest to peak exercise. In each patient with AR who had a normal LV end-systolic pressure-volume slope, the exercise LV ejection fraction increased six ejection fraction 
units, whereas those patients with a reduced endsystolic pressure-volume slope had a decrease in their LV ejection fraction of five ejection fraction units. Subsequently, Shen et al. ${ }^{7}$ demonstrated that the change in radionuclide $L V$ ejection fraction from rest to peak exercise in control and AR groups was linearly related to the slope of the $\mathrm{LV}$ end-systolic pressure-volume relationship. These investigators suggested that the change in radionuclide LV ejection fraction from rest to peak exercise might be related to the long-term effects of chronic, severe AR on LV contractile performance.

In contrast to these observations, other investigators have suggested that the radionuclide $L V$ ejection fraction response to exercise in patients with AR may be determined by resting hemodynamic conditions. ${ }^{19,}{ }^{20}$ For example, Massie et al. ${ }^{19}$ reported that the change in radionuclide $L V$ ejection fraction from rest to peak exercise was inversely related to pulmonary capillary wedge pressure $(r=-0.69, p<0.01)$. In addition, Boucher et al. ${ }^{20}$ noted that a peak exercise pulmonary capillary wedge pressure of $15 \mathrm{~mm} \mathrm{Hg}$ or more identified patients with AR who had lower radionuclide $\mathrm{LV}$ ejection fractions $(p<0.01)$ and higher end-systolic volumes at peak exercise $(p<0.01)$, compared to patients with AR who had more normal pulmonary capillary wedge pressures at peak exercise. Still other investigators have suggested that there is a relationship between the change in radionuclide $L V$ ejection fraction from rest to peak exercise and LV stress, ${ }^{21}$ and the change in LVESV, ${ }^{22}$ cardiac output, ${ }^{23}$ or systemic vascular resistance. ${ }^{6}$ An increase in the radionuclide $L V$ ejection fraction in patients with $\mathrm{AR}$ might therefore be associated with a lower resting $L V$ peak or end-systolic stress, a reduction in systemic vascular resistance, regurgitant index, and LVESV, and an improvement in cardiac output with exercise. Consequently, several hemodynamic mechanisms may be operative in patients with chronic, severe AR in determining how the left ventricle responds to the stress of exercise.

The data from the present investigation expands on these observations. It indicates that depending on whether the peak exercise LV ejection fraction or the change in $L V$ ejection fraction from rest to peak exercise is used to evaluatc $L V$ performance, the hemodynamic determinants of these measures may differ in patients with AR. Notably, the peak exercise radionuclide $L V$ ejection fraction was principally related to $\mathrm{LV}$ chamber performance, but the change in $\mathrm{LV}$ ejection fraction from rest to peak exercise was related to the corresponding change in peripheral vascular responses (i.e., systemic vascular resistance) and the resultant change in regurgitant index and LV volumes. Consequently, these data suggest that $\mathrm{LV}$ chamber performance might be the principal determinant of the peak exercise radionuclide $L V$ ejection fraction, and that the change in LV ejection fraction from rest to peak exercise might be more affected by the response of the peripheral vasculature to chronic, severe $\mathrm{LV}$ volume overload.

Establishing the physiologic determinants of the peak exercise radionuclide $L V$ ejection fraction or the change in LV ejection fraction from rest to peak exercise is necessary to understand how these data might contribute to the selection of patients with $A R$ for cardiac catheterization and possible aortic valve surgery. It has been suggested that there is a natural hemodynamic progression in patients with chronic, severe volume overload that manifests initially as compensated, eccentric LV hypertrophy followed by $\mathrm{LV}$ systolic dysfunction in the absence of myocardial dysfunction and, finally, by the supervention of myocardial dysfunction. ${ }^{24}$ We have previously shown that LV chamber elastance, (i.e., $E_{\max }$ ) may be abnormal in patients with AR in the absence of myocardial dysfunction. In those patients with $\mathrm{AR}$ who have an abnormal $\mathrm{E}_{\max }$, the $\mathrm{LV}$ volume-to-mass ratio was increased, suggesting that inadequate, eccentric LV hypertrophy had developed and that LV shape had been altered. ${ }^{25}$ If the preload reserve of these patients is fully utilized, then an increase in LV load, whether it be induced pharmacologically or by exercise, might equate to either no change or a reduction in shortening, in comparison to patients with $A R$ and normal $E_{\max }$ in whom these adaptive hemodynamic processes have presumably not been exhausted. Because the peak exercise radionuclide $\mathrm{LV}$ ejection fraction incorporates both the long-term effects of chronic, scvere LV volume overload on myocardial performance and these other detrimental hemodynamic effects, it is not surprising that $\mathrm{E}_{\mathrm{max}}$ is a major determinant of the peak exercise radionuclide LV ejection fraction.

Our data would also suggest that each patient with AR who has a peak exercise $L V$ ejection fraction of less than $50 \%$ has abnormal LV chamber performance. This might be especially important in patients with $\mathrm{AR}$ who have no symptoms, in whom noninvasive techniques are commonly used to characterize sequential changes in LV systolic performance. Progression from a peak exercise $L V$ ejection fraction of $50 \%$ or more to one that is less than $50 \%$ might be an early marker of those patients with $A R$ who have progressed into a new hemodynamic phase in which LV chamber performance is impaired. Sequential changes in the peak exercise LV ejection fraction are particularly important, because an isolated peak exercise LV ejection fraction of less than $50 \%$ may represent LV systolic dysfunction due to 
the existence of inadequate, eccentric LV hypertrophy and alterations of LV shape,$^{22}$ myocardial dysfunction, ${ }^{7}$ or both. When these data are coupled with the recent observations of Siemienczuk et al., ${ }^{3}$ a hemodynamic explanation for the clinical and prognostic significance of an $\mathrm{LV}$ ejection fraction at peak exercise of $50 \%$ or less in patients with AR who have minimal or no symptoms can be appreciated.

The observation that the response of the peripheral vasculature to exercise may be an important determinant of the change in $\mathrm{LV}$ ejection fraction from rest to peak exercise in patients with AR substantiates the observations of other investigators. ${ }^{19-23} \mathrm{~A}$ reduction in systemic vascular resistance due to exercise, as observed in this investigation, produced a corollary reduction in regurgitant index and therefore a maintenance or improvement in $\mathrm{LV}$ ejection fraction from rest to peak exercise. The change in $L V$ ejection fraction from rest to peak exercise appears to represent, therefore, the effects of chronic, severe volume overload on peripheral vascular adaptations. Recently, Borow et al. ${ }^{26}$ demonstrated that resting myocardial performance and myocardial reserve may be preserved in the type I diabetic group whether or not they have a normal increase from rest to peak exercise in radionuclide $L V$ ejection fraction or if they have no change or a decrement in LV ejection fraction with exercise. Consequently, a change in radionuclide $L V$ ejection fraction from rest to peak exercise in patients with AR should probably not be equated with the presence or absence of abnormal myocardial performance at rest or the adequacy of myocardial reserve, but whether or not maladaptions of the peripheral vasculature have developed with the duration of chronic, severe AR. These data add further support to the important influence of the duration of chronic, severe $\mathrm{AR}$ on exercise performance and functional outcome after aortic valve surgery reported by Bonow et al. ${ }^{27}$

In this investigation, we used supine bicycle exercise to assess the changes in radionuclide $L V$ volumes and ejection fractions. The response of the LV ejection fraction should be comparable whether upright or supine bicycle exercise is used..$^{28}$ In general, the average response of the LVEDV and LVESV and LV ejection fractions in our patients with AR was comparable to those previously reported by other investigators ${ }^{1,2,5-7,18-22,29}$; that is, all of the control patients demonstrated an appropriate increase in $\mathrm{LV}$ ejection fraction, while five of 21 patients with AR had an increase in LV ejection fraction. The remaining patients had either no change or a decrease in ejection fraction with exercise. The data in this investigation should therefore be comparable to previous studies in which supine bicycle exercise was used to assess the LV ejection fraction response to exercise in patients with AR. ${ }^{5,6,19,22}$

We also used radionuclide angiography to calculate multiple hemodynamic parameters at rest and during peak exercise to compare with the radionuclide LV ejection fraction response to exercise. We have demonstrated that $\mathrm{LV}$ volumes and ejection fractions can be obtained accurately with the use of an attenuation correction technique previously validated in this laboratory. ${ }^{9}$ Moreover, we employed a modification of the technique of Maddahi et al. ${ }^{10}$ to obtain right ventricular end-diastolic and end-systolic counts and ejection fraction. The LV regurgitant index, "effective" LV stroke volume and cardiac output, and system vascular resistance were then calculated both at rest and peak exercise. Importantly, we have used this approach to calculate right ventricular volumes for comparison to biplane cineventriculographic right ventricular volumes ${ }^{11}$ and to diagnosis and assess therapy of right ventricular infarction. ${ }^{12}$ The term effective should be clarified. We defined effective LV stroke volume and cardiac output as the beat and minute output, respectively, used to perfuse the peripheral vascular tissues. Consequently, total LV stroke or cardiac output was decremented by the regurgitant volume to calculate these hemodynamic parameters. 'The cardiac output calculated on the day before cardiac catheterization with the use of this radionuclide approach was similar to that obtained with the use of the thermodilution technique, suggesting that this approach should have provided reliable information about right and left ventricular performance, cardiac output, and systemic vascular resistance.

In conclusion, the data in this investigation suggest that the radionuclide $L V$ ejection fraction during peak exercise is most strongly affected by LV cham. ber performance, and that the change in LV ejection fraction from rest to peak exercise is principally determined by peripheral vascular responses and their resultant effects on regurgitant index and LV volumes. Consequently, because the peak exercise LV ejection fraction reflects $L V$ chamber performance and it has been shown to predict symptomatic deterioration in patients with $\mathrm{AR},{ }^{3}$ it might be an appropriate noninvasive exercise radionuclide parameter to follow for establishing the time period for invasive studies and possible aortic valve replacement.

We appreciate the assistance of Christina Brown, BS, Janet Petrusha, RN, Jacqueline Anderson, and Jacqueline LeRoy in the preparation of this manuscript. 


\section{REFERENCES}

1. Borer JA, Bacharach SL, Green MV, et al. Exercise induced left ventricular dysfunction in symptomatic and asymptomatic patient with aortic regurgitation: assessment with radionuclide cineangiography. Am J Cardiol 1978;42:351-7.

2. Dehmer GJ, Firth BG, Hillis LD, et al. Alterations in left ventricular volumes and ejection fraction at rest and during exercise in patients with aortic regurgitation. Am J Cardiol 1981; 48:17-27.

3. Siemienczuk D, Greenburg BH, Morris C, et al. Chronic aortic insufficiency: factors associated with progression to aortic valve replacement. Ann Intern Med 1989;110:587-92.

4. Iskandrian AS, Heo J. Radionuclide angiographic evaluation of left ventricular performance at rest and during exercise in patients with aortic regurgitation. AM HEART J 1986; 111:1143-9.

5. Goldman ME, Packer M, Horowitz SF, et al. Relation between exercise-induced changes in ejection fraction and systolic loading conditions at rest in aortic regurgitation. J Am Coll Cardiol 1984;3:924-9.

6. Kawanishi DT, McKay CR, Chandraratna AN, et al. Cardiovascular response to dynamic exercise in patients with chronic symptomatic mild-to-moderate and severe aortic regurgitation. Circulation 1986;73:62-72.

7. Shen WF, Roubin GS, Choong CY, et al. Evaluation of relationship between myocardial contractile state and left ventricular function in patients with aortic regurgitation. Circulation 1985;71:31-8.

8. Starling MR, Walsh RA, Dell'Italia LJ, Mancini GBJ, Lasher JC, Lancaster JL. The relationship of various measures of end-systole to the left ventricular maximum time-varying elastance in man. Circulation 1987;76:32-13.

9. Starling MR, Dell'Italia LJ, Walsh RA, Little WC, Benedetto AR, Nusynowitz ML. Accurate estimates of absolute left ventricular volumes from equilibrium radionuclide angiographic count data using a simple geometric attenuation correction. J Am Coll Cardiol 1984;3:789-98.

10. Maddahi J, Berman DS, Matsuoka DT, et al. A new technique for assessing right ventricular ejection fraction using rapid multiple-gated equilibium cardiac blood pool scintigraphy. Circulation 1979;60:581-9.

11. Dell'Italia LJ, Starling MR, Walsh RA, Badke FR, Lasher JC, Blumhardt R. Validation of attenuation-corrected equilibrium radionuclide angiographic determinations of right ventricular volume: comparison with cast-validated biplane cineventriculography. Circulation 1985;72:317-26.

12. Dell'Italia LJ, Starling MR, Crawford MH, Boros BL, Chaudhuri TK, O'Rourke RA. Right ventricular infarction: identification by hemodynamic measurements before and after volume loading and correlations with noninvasive techniques. $J$ Am Coll Cardiol 1984;4:931-9.

13. Sagawa K, Suga H, Shoukas AA, Bakalas K. End-systolic pressure-volume ratio: a new index of contractility. Am J Cardiol 1979;40:748-53.

14. Sagawa K: The ventricular pressure-volume diagram revisited. Circ Res 1978;43:677-87.
15. Sagawa K: The end-systolic pressure-volume diagram revisited. Circulation 1981;63:1223-7.

16. Maughan WL, Sunagawa K, Burkhoff D, Sagawa K. Effect of arterial impedance changes on the end-systolic pressure-volume relationship. Circ Res 1984;54:595-602.

17. Suga H, Hisano K, Goto $Y$, Yamanda $O$. Normalization of end-systolic pressure-volume relationship and $\mathrm{E}_{\max }$ of different heart sizes. Jpn Circ J 1984;48:136-43.

18. Schuler G, Von Olshausen K, Schwarz F, et al. Non-invasive assessment of myocardial contractility in asymptomatic patients with severe aortic regurgitation and normal left ventricular ejection fraction at rest. Am J Cardiol 1982;50:45-52.

19. Massie BM, Kramer BL, Loge D, et al. Ejection fraction response to supine exercise in asymptomatic aortic regurgitation: relation to simultaneous hemodynamic measurements. J Am Coll Cardiol 1985;5:847-55.

20. Boucher CA, Wilson RA, Kanarek DJ, et al. Exercise testing in asymptomatic or minimally symptomatic aortic regurgitation: relationship of left ventricular ejection fraction to left ventricular filling pressure during exercise. Circulation 1983;67:1091-9.

21. Shen WF, Fletcher PJ, Roubin GS, Harris PJ, Kelly DT. Relation between left ventricular functional reserve during exercise and resting systolic loading conditions in chronic aortic regurgitation. Am J Cardiol 1985;58:757-61.

22. Greenburg RH, Massie RM, Thomas D, et al. Association between exercise ejection fraction response and systolic wall stress in patients with chronic aortic insufficiency. Circulation 1985;71:458-65.

23. Osbakken M, Bove AA, Spann JF. Left ventricular function in chronic aortic regurgitation with reference to end-systolic pressure, volume and stress relations. Am J Cardiol 1981; 47:193-8.

24. Ross J. Afterload mismatch in aortic and mitral valve disease: implications for surgical therapy. J Am Coll Cardiol 1985;5:81126.

25. Starling R, Kirsh MM, Montgomery DG, Gross MD. Mechanisins for left ventricular systolic dysfunction in aortic regurgitation: importance for predicting the functional response to aortic valve replacement. J Am Coll Cardiol 1991;17:887-97.

26. Borow KM, Jaspan JB, Williams KA, Neumann A, WolinskiWalley $P$, Lange RM. Myocardial mechanics in young adult patients with diabetes mellitus: effects of altered load, inotropic state and dynamic exercise. J Am Coll Cardiol 1990;15:150817.

27. Bonow RO, Rosing DR, Maron BJ. Reversal of left ventricular dysfunction after aortic valve replacement for chronic aortic regurgitation: influence of duration of preoperative left ventricular dysfunction. Circulation 1984;70:570-9.

28. Crawford M, White DH, Amon KW. Echocardiographic evaluation of left ventricular size and performance during handgrip and supine and upright bicycle exercise. Circulation 1979;59:1188-96.

29. Higginbotham MB, Morris KG, Coleman RE, Cobb FR. Sexrelated differences in the normal cardiac response to upright exercise. Circulation 1984;70:357-66. 